\title{
Permittivity of lossy composite materials
}

Cite as: Journal of Applied Physics 83, 425 (1998); https://doi.org/10.1063/1.366725

Submitted: 03 June 1997. Accepted: 19 September 1997 . Published Online: 04 June 1998

A. Boudida, A. Beroual, and C. Brosseau

\section{ARTICLES YOU MAY BE INTERESTED IN}

Complex effective permittivity of a lossy composite material

Journal of Applied Physics 80, 4560 (1996); https://doi.org/10.1063/1.363438

How do shape anisotropy and spatial orientation of the constituents affect the permittivity of dielectric heterostructures?

Journal of Applied Physics 88, 7278 (2000); https://doi.org/10.1063/1.1321779

Electrical conductivity in inhomogeneous media

AIP Conference Proceedings 40, 2 (1978); https://doi.org/10.1063/1.31150
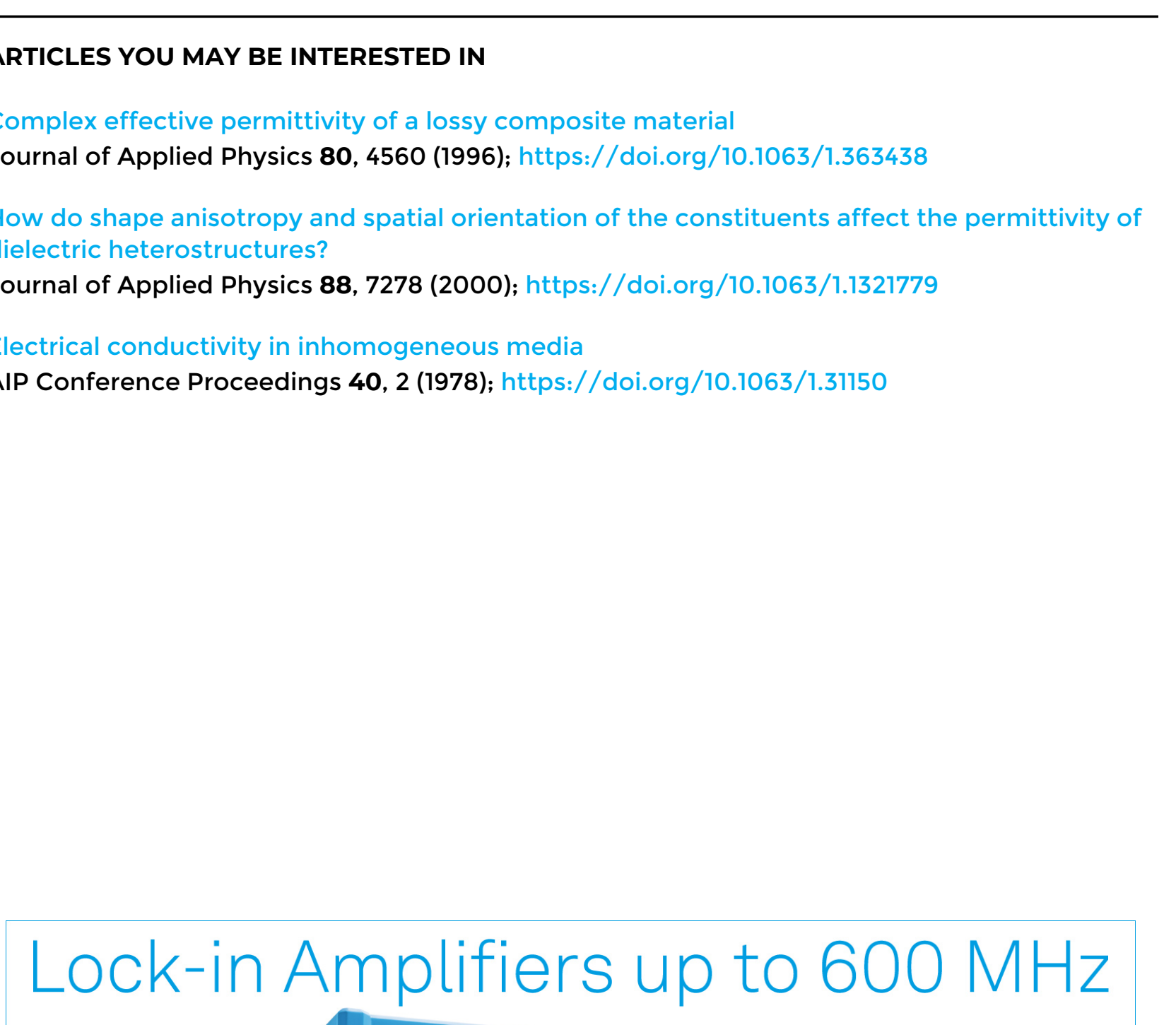

starting at

$\$ 6,210$

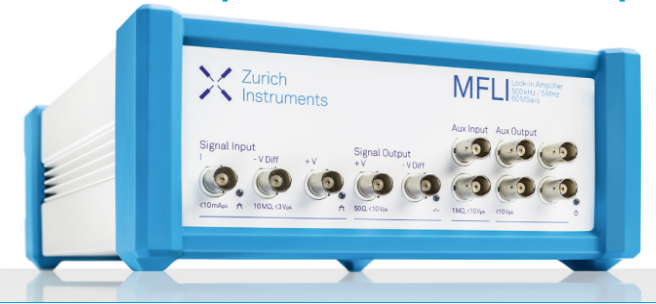

Journal of Applied Physics 83, 425 (1998); https://doi.org/10.1063/1.366725

(c) 1998 American Institute of Physics.

83, 425 


\title{
Permittivity of lossy composite materials
}

\author{
A. Boudida and A. Berouala)

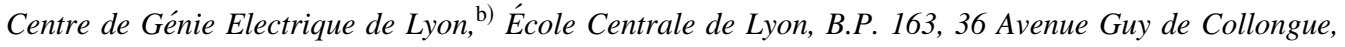 \\ 69131 Ecully Cedex, France \\ C. Brosseau ${ }^{\text {) }}$ \\ Département de Physique, Laboratoire d'Étude des Matériaux, Université de Bretagne Occidentale, \\ 6 avenue Le Gorgeu, B.P. 809, 29285 Brest Cedex, France
}

(Received 3 June 1997; accepted for publication 19 September 1997)

\begin{abstract}
An $a b$ initio numerical simulation model has been used to compute the complex effective dielectric constant of a two-component lossy composite material, in the quasistatic limit. A computational algorithm with a conventional finite element formulation solves Laplace's equation for a spatially heterogeneous medium, using the field calculation package FLUX3D. In this way, different three-dimensional topological arrangements of the components were considered. The composite material consists of dense spheres of uniform size that are arranged in simple, body-centered, and face-centered cubic lattices. The accuracy of the method is checked by comparing with results previously presented in the literature. Detailed predictions provide a comparison with percolation theory when the imaginary part of the relative permittivity of the spheres is very large. A comparison with McLachlan's generalized effective medium equation [D. S. McLachlan, J. Phys. C 20, 865 (1987)] is further provided over a wide range of conditions. From these calculations one can conclude that there are significant discrepancies between the $a b$ initio evaluated values of the effective permittivity and those obtained on the basis of McLachlan's analysis. On the one hand, the numerical method demonstrated here shows that the real part of the effective permittivity, obtained from $a b$ initio results, can be significantly different from that predicted on the basis of McLachlan's equation when the imaginary part of the permittivity of the inclusion is very large compared to its real part. On the other hand, these computational results capture the trends in the percolation threshold variation with cubic lattice packing. We measured the exponents $s$ and $t$ which determine how the real and imaginary parts of the permittivity scales with the distance from the percolation threshold. This behavior is most probably due to the drastic differences in the basic assumptions existing between McLachlan's modeling and our numerical approach. In particular, this analysis makes it clear that any approach based only on the dipole approximation must fail to correctly describe the complex effective dielectric constant, over the entire range of volume fraction of spherical inclusions. (C) 1998 American Institute of Physics. [S0021-8979(98)00101-7]
\end{abstract}

\section{INTRODUCTION}

The study of dielectric properties of heterostructures has recently seen an enhanced level of activity, prompted in part by the insight such investigations might give in a number of practical applications in the electronics and aerospace industries, to name but a few. Extensive research work on this subject has been reported in the literature during the past several years. The general state of the art has been reviewed by Landauer ${ }^{1}$ and more recently by Priou. ${ }^{2}$ On the theory side, these studies have also fundamental consequences in connection with the problem of localization of waves, i.e., dielectric structures with photonic band gaps. ${ }^{3}$ On the practical side, recent works indicate it is possible that the construction of composite materials may constitute a useful method for obtaining materials with desirable specific properties, e.g., composite superconductors, ${ }^{4}$ nanostructured components. ${ }^{2}$ The microstructure morphology of these mate-

\footnotetext{
a) Tele: (33) 04721861 10; Fax: (33) 04784337 17; Electronic mail: beroual@ trotek.ec-lyon.fr

b)UPRESA CNRS 5005 .

c) Tele: (33) 02980161 05; Fax (33) 02980161 31; Electronic mail: brosseau@univ-brest.fr
}

rials clearly plays a major role in determining their effective dielectric properties, and an understanding of the behavior of the complex permittivity is essential to establish which properties of the composites might follow simply from their geometry.

One fundamental issue that has driven many of the experiments on electromagnetic properties of heterogeneous media is the nature of the conductivity- and permittivityconcentration characteristics. In order to fix notation and to give an account of the earlier work, we begin by recalling some results that we will need, connected to the percolation analysis of insulator-metal composite systems. The percolation model gave rise to a number of applications to explain the behavior of a variety of condensed-matter systems and several generalizations are documented in a series of recent reviews and books. ${ }^{5,6}$ The percolation process is geometrical in nature and provides a theoretical framework for understanding collective phenomena in a number of systems with quenched disorder. For insulator-conductor mixtures there exist a wide variety of predictions in the literature that show a critical behavior near a concentration threshold $f_{c}$ of the conducting material. A number of researchers have obtained power-law expressions for the dc electrical conductivity and 
TABLE I. Critical parameters for bond and site percolation models on cubic lattices.

\begin{tabular}{|c|c|c|c|c|}
\hline Lattice & $\begin{array}{c}\text { Critical } \\
\text { (percolation } \\
\text { threshold) } \\
\text { bond } \\
\text { probability }\end{array}$ & $\begin{array}{c}\text { Critical } \\
\text { (percolation } \\
\text { threshold) } \\
\text { site } \\
\text { probability }\end{array}$ & $\begin{array}{c}\text { Coordination } \\
\text { number }\end{array}$ & $\begin{array}{l}\text { Packing } \\
\text { fraction }\end{array}$ \\
\hline Simple cubic $(\mathrm{sc})$ & 0.249 & 0.312 & 6 & 0.524 \\
\hline Body-centered cubic (bcc) & 0.180 & 0.246 & 8 & 0.680 \\
\hline Face-centered cubic (fcc) & 0.119 & 0.198 & 12 & 0.741 \\
\hline
\end{tabular}

have shown that it is nonzero above $f_{c}$ and obeys a scaling relation $\left(f-f_{c}\right)^{t}$ while the real part of the permittivity shows a critical behavior $\sim\left|f-f_{c}\right|^{-s}$ on the insulating side (below $f_{c}$ ), where $f$ denotes the volume fraction of the high conductivity component. Furthermore, the critical exponents $t$ and $s$ depend only on the dimensionality of the system and not on the microscopic details of the system, i.e., for threedimensional systems $t \cong 1.5-2$ and $s \cong 0.7 .^{7-9}$ This picture has been developed by the concerted use of exact analytical theories and numerical simulations. While the exponents $s$ and $t$ are universal, the percolation fraction $f_{c}$ depends on the geometrical details of the system. This behavior corresponds to the formation of infinitely large connected clusters of conducting elements, creating a connection between the electrodes, i.e., the so-called " percolation path." For a rigid, periodic cubic array (built of sites or bonds) of conducting spheres, the critical (percolation threshold) probability depends on the topological arrangement of the spheres. Table I, abstracted in part from Ref. 5 reveals the tendency of these probabilities to decrease as the coordination number (packing fraction) is increased. Recently, there have been many experimental verifications of the values of the scaling exponents predicted by the percolation model for a variety of composite materials. $^{10-14}$

Early theoretical attempts to model the dielectric properties of a two-component composite material consisting of inclusions of constituent 1 (with permittivity $\epsilon_{1}$ ) surrounded by a background material of constituent 2 (with permittivity $\epsilon_{2}$ ) relied primarily on mixing law calculations. ${ }^{1,2,15-17}$ Much of it has centered around the expression of the permittivity of a heterogeneous material in terms of the permittivities of the different components and using more or less detailed information of the particle distribution. These semiempirical methods are especially tied to the specific materials they address and are difficult to generalize. Moreover the mixing formulas all give more or less the same results at low concentration of inclusions (dilute limit). ${ }^{18,19}$ When they were originally formulated, these mixing laws were intended for static conditions. In practice, many calculations in the past decade have demonstrated that these formulas give decent results for the nonzero frequency domain but are unable to reproduce a number of experimental features such as a proper description of the local field distribution at high volume loading and the evaluation of the percolation threshold. Typical mixture formulas which have generated a great deal of interest are those of Maxwell Garnett and of Bruggeman. ${ }^{1}$ If the two components of the composite material are metal and insulator the former does not yield a percolation thresh- old at any finite values of $f$, whereas the latter predicts a percolation threshold of $f_{c}=0.33$ for dense spherical inclusions.

McLachlan postulated a generalized effective medium equation for describing the dielectric properties, in the quasistatic limit, of a binary mixture and found remarkable singularities of the real part of permittivity near the percolation threshold $f_{c}$ where the imaginary part diverges. ${ }^{20}$ This equation, developed heuristically, was originally written in terms of the conductivity $\sigma$, as

$$
\frac{f\left(\sigma_{1}^{1 / t}-\sigma^{1 / t}\right)}{\sigma_{1}^{1 / t}+A \sigma^{1 / t}}+\frac{(1-f)\left(\sigma_{2}^{1 / t}-\sigma^{1 / t}\right)}{\sigma_{2}^{1 / t}+A \sigma^{1 / t}}=0,
$$

where $\sigma_{1}$ (resp. $\sigma_{2}$ ) is the conductivity of the more conducting (resp. insulating) component and $A \equiv\left(1-f_{c}\right) / f_{c}$. Reference is made to McLachlan for the full details. ${ }^{20,21}$ Since McLachlan phenomenological analysis does not resort to restrictive or presumptive assumptions on the spatial distribution and particle shapes, it ignores almost all the details of the microgeometry and is therefore applicable to quite general heterogeneous systems including those with a periodic geometry. Note that Eq. (1) reduces to the Bruggeman symmetric equation by setting $t=1$ and to the Bruggeman asymmetric equation when $\sigma_{2}=0$ and $f_{c}=0$ or when $\sigma_{1} \rightarrow \infty$ and $f_{c}=1$. In many cases this phenomenological equation give results surprisingly close to the mark, e.g., microemulsion of AOT, (sodium di-2-ethylhexylsulfosuccinate), water and isooctane. $^{20,21}$ However two main difficulties, to which we return below, are worth noting. First, since McLachlan's equation is rooted in the Bruggeman theory, it cannot realistically describe the permittivity at large concentrations of inclusions because this effective medium parametrization includes only dipolar effects and does not include quadrupole or higher interactions. Second, there is no reference in this equation to the microstructure geometry of the constituents in the system.

Below, we present the results from an ab initio numerical study of the complex effective permittivity of a twocomponent periodic composite material. The present numerical experiments aimed at comparing our results to the analytical data of McLachlan over a wide range of conditions. Such a comparison is of interest as it may provide insight concerning the polarizability properties of inclusions. Additional motivation for this study is to complement earlier work on lossy composite materials. ${ }^{22}$

The content of this manuscript is organized as follows. Section II will be devoted to a brief description of some of 
the essential features of our computational procedure. Next Sec. III presents the detailed results of the different numerical evaluations of the effective complex permittivity for a variety of rigid, periodic arrays of inclusions. Our results are compared with those obtained from McLachlan's phenomenological equation. In the concluding remarks, Sec. IV, we suggest future directions for continuing the development of the research.

\section{SIMULATION TECHNIQUE}

An extensive literature exists on the numerical evaluation of the dielectric properties of heterostructures (see Refs. 1 and 15-17 for surveys and comprehensive reference lists). A full study of the effective properties of random composite media is a formidable task but, in fact, certain simplifications may be introduced which allow essential features to be isolated. With recent advances in numerical simulation, it is now possible to obtain high-precision values of the effective dielectric constant of multicomponent composite materials. Besides providing clues to the understanding of complex systems, computer simulations are also assumed to give answers which do not differ in a marked way from the ones one would find by performing experiments on real systems.

In a previous article from our team, the effective dielectric constant of a two-dimensional (or three-dimensional axisymmetric) lossy composite material in the quasistatic limit was considered ${ }^{22}$ and a comparison of the results of simulations with the predictions of analytical theories was provided, e.g., Bergman and Milton analysis. ${ }^{9,23}$ The conclusion of this study was that this simulation accounts quantitatively for the evaluation of the complex permittivity of these materials. As noted before, the method is quasistatic. For this reason, the wave cannot resolve the individual inhomogeneities and the dielectric properties of the composite can be defined by a single effective permittivity.

The simulations presented in this article are based on the resolution of a finite element (FE) modeling using the field calculation package FLUX3D. Before proceeding further, it is necessary to begin with a discussion of the computational method. Our code has been developed over a number of years. ${ }^{24,25}$ Since the attributes of the algorithm for studying two-component heterogenous materials have already been described in detail elsewhere, ${ }^{22,24,25}$ we shall touch only briefly on its main features. Briefly they are: (1) The general features of our results do not depend on the effective medium approximation. Our systematic approach avoids the somewhat ad hoc manner in which mixture equations are often introduced in discussions of dielectric properties of composite materials. (2) This method is exact for regulars arrays forming a crystal lattice but we have to make approximations when applying it to random media since an exact calculation of higher multipole interactions is intractable in that case. (3) The method includes all multipoles exactly: it has proven very successful for high permittivity contrast ratios because the response of the system to a potential is found from coupled multipole equations (multipole polarizabilities) which are not contained in simple dipole mixture rules or mean-field approaches. (4) This calculational method is not computationally intensive. The computational

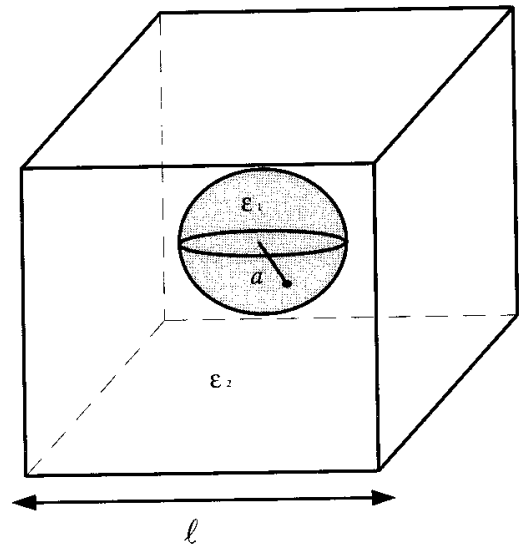

FIG. 1. Notation relating to the unit cell of the two-component periodic composite material investigated in the finite element model computation. The spherical inclusion with dielectric constant $\epsilon_{1}$ is periodically arranged in a three-dimensional cubic lattice structure. The dielectric constant of the remaining space is $\epsilon_{2}$.

time taken to run our simulations on a HP model 712/80 workstation depends upon the quality of the meshing of about 3500 equations and is $8 \mathrm{~min}$ for calculating the permittivity of a typical configuration. This is an important consideration to care about if we want to study the influence of randomness on the dielectric properties of heterostructures. ${ }^{25-27}$

In this study we consider a composite medium consisting of two materials having relative permittivities $\epsilon_{1}$ and $\epsilon_{2}$ with respect to the vacuum permittivity. We begin our analysis by refering to Fig. 1 which shows a unit cell of a simple cubic periodic structure. We assume, in the following, that all the lengths $(l, a)$ are dimensionless and that the side of the cell has the specific value $l=2$. In the numerical experiments of Sec. III, the component 1 (here, spherical inclusions of uniform size) is a lossy material, i.e., $\epsilon_{1}=\epsilon_{1}^{\prime}-i \epsilon_{1}^{\prime \prime}$, and the component 2 (host) is a pure dielectric, i.e., $\epsilon_{2}=\epsilon_{2}^{\prime}$. Details of the simulation procedure can be found in previous work. ${ }^{22,24,25}$

The method has been numerically verified for a variety of benchmarks. ${ }^{22,25}$ We include such test below for demonstration purpose. In this test problem, results are given for a simple cubic configuration using $\epsilon_{1}=38.2-i 22.1$ and $\epsilon_{2}$ $=1.0-i 0.0$. These values were chosen for the purpose of comparison with the results of Calame et al. ${ }^{28}$ The $a b$ initio results are graphed in Figs. 2(a) and 2(b). The solutions compare very well with the data of Calame et al. It is interesting to note, that in common with the ab initio results, the behavior of the permittivity is dramatically altered for a volume fraction $f \cong 0.5$ which is close to the percolation threshold, corresponding to the touching spheres condition, for an infinite simple cubic lattice of uniform size spheres (see Table I). The satisfactory overall agreement of our computational model with the numerical results of Calame et al. along with previous comparison with analytical theories ${ }^{9,23}$ gives us confidence that our simulation will give a reliable description of the effective permittivity of lossy composite materials. 

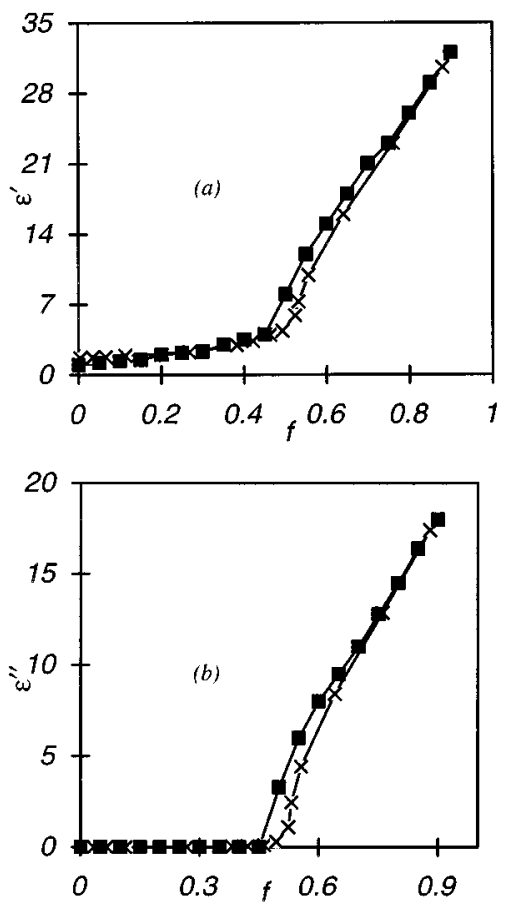

FIG. 2. (a) The real part $(X)$ of the effective permittivity is shown as a function of the volume fraction $f$ of the inclusion phase. Spherical inclusions (permittivity $\epsilon_{1}=38.2-i 22.1$ ) are placed in a host matrix material of permittivity $\epsilon_{2}=1.0-i 0.0$. Simple cubic lattice. Also shown is the Calame et al. (Ref. 28) prediction (square). (b) The imaginary part $(\times)$ of the effective permittivity is shown as a function of the volume fraction $f$ of the inclusion phase. Spherical inclusions (permittivity $\epsilon_{1}=38.2-i 22.1$ ) are placed in a host matrix material of permittivity $\epsilon_{2}=1.0-i 0.0$. Simple cubic lattice. Also shown is the Calame et al. (Ref. 28) prediction (square).

The machinery for calculating the complex effective permittivity presented in this article is now in place. In the next section the relevant details of the results are described and discussed.

\section{RESULTS AND DISCUSSION}

Through the use of the numerical technique described in the previous section, a set of different simulations corresponding to selected three-dimensional geometries were performed and are discussed in detail. Specifically we present numerical results for several regular arrays of spherical inclusions of uniform size forming a crystal lattice in a host matrix. Three types of structures are investigated: simple (sc), body-centered (bcc), and face-centered (fcc) cubic lattices.

\section{A. Comparison of numerical predictions with McLachlan analysis}

We begin this presentation by considering a system in which spheres are spatially distributed at the nodes of a simple cubic lattice. Two sets of permittivity component values were studied: $\epsilon_{1}=80-i 10^{6}, \epsilon_{2}=2-i 0$ and $\epsilon_{1}=80-i 10^{4}$, $\epsilon_{2}=2-i 0$. In either case the sphere to host real part of permittivity is 40 . Figures 3 and 4 compare the simulation data of the complex effective permittivity with predictions from McLachlan's equation. ${ }^{20}$ As can be seen clearly from these
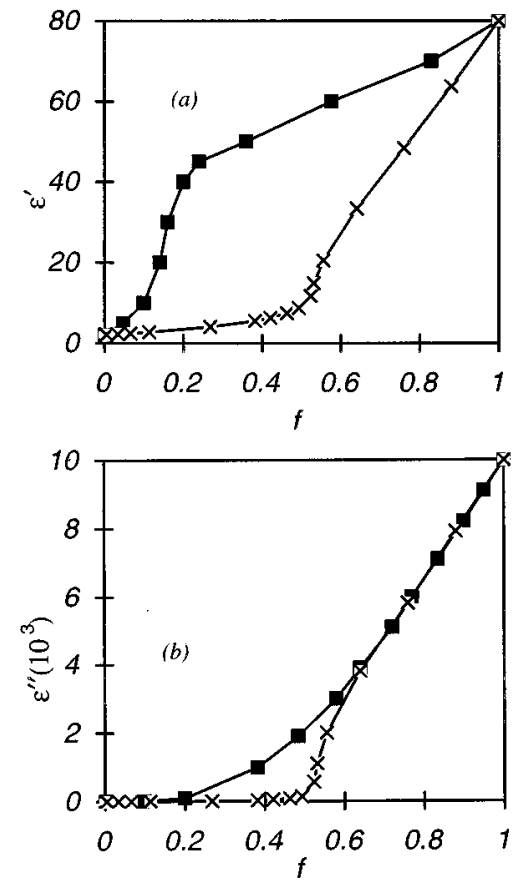

FIG. 3. (a) The real part $(X)$ of the effective permittivity is shown as a function of the volume fraction $f$ of the inclusion phase. Inclusions (permittivity $\epsilon_{1}=80-i 10^{4}$ ) are placed in a host matrix material of permittivity $\epsilon_{2}$ $=2-i 0.0$. Simple cubic lattice. Squares: prediction from McLachlan equation (Ref. 20). (b) The imaginary part $(X)$ of the effective permittivity is shown as a function of the volume fraction $f$ of the inclusion phase. Inclusions (permittivity $\epsilon_{1}=80-i 10^{4}$ ) are placed in a host matrix material of permittivity $\epsilon_{2}=2-i 0.0$. Simple cubic lattice. Squares: prediction from McLachlan equation (Ref. 20).

figures, the values of $\epsilon^{\prime}$ and $\epsilon^{\prime \prime}$ given here deviate markedly with the published results of McLachlan. For these two sets of parameters, the simulation data predict a lower real (and imaginary) part than the McLachlan analysis. The most distinguishing feature of McLachlan's data is the sharp increase in permittivity with volume fraction $f$ which is particularly evident in the real part for $f \leqslant 0.2$ [Figs. 3(a) and 4(a)]. We observe that the real part of the effective permittivity increases rapidly for $\epsilon_{1}=80-i 10^{6}$ [Fig. 4(a)] and slowly for $\epsilon_{1}=80-i 10^{4}$ [Fig. 3(a)] but in both cases the values of $\epsilon^{\prime}$ remain moderate up to the close packing limit. Computer simulations produce a result of quite different form and magnitude over the entire range of volume fraction. The largest deviation appears for the higher value of $\epsilon_{1}^{\prime \prime}$. Departures from the dilute limit appear also at $f \cong 0.1$ for the imaginary part of the permittivity. We observe that the results superimpose rather nicely at the higher volume fractions, i.e., $f$ $>0.6$.

The differences between the two approaches are also striking in the transition region when the spheres make contact. The present analysis and that of McLachlan both predict a critical volume fraction of inclusions $f_{c}$ where $\epsilon^{\prime \prime}$ begins deviating substantially from zero. We estimate $f_{c}=0.50$ \pm 0.02 . for our results while McLachlan's equation uses as input $f_{c}=0.16,{ }^{29}$ i.e., well below the close packing limit concentration which is close to the touching spheres condition in the sc structure (Table I). We note also that the critical region 

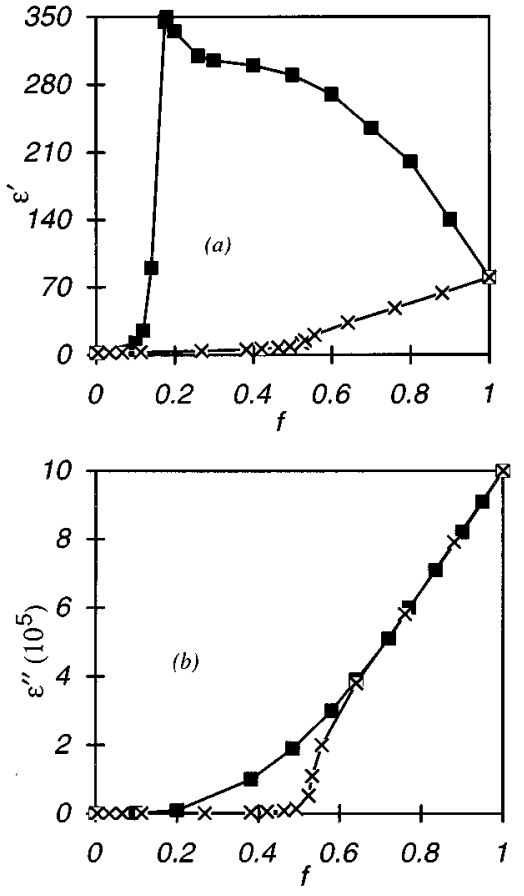

FIG. 4. (a) The real part $(X)$ of the effective permittivity is shown as a function of the volume fraction $f$ of the inclusion phase. Inclusions (permittivity $\epsilon_{1}=80-i 10^{6}$ ) are placed in a host matrix material of permittivity $\epsilon_{2}$ $=2-i 0.0$. Simple cubic lattice. Squares: prediction from McLachlan equation (Ref. 20). (b) The imaginary part ( $X$ ) of the effective permittivity is shown as a function of the volume fraction $f$ of the inclusion phase. Inclusions (permittivity $\epsilon_{1}=80-i 10^{6}$ ) are placed in a host matrix material of permittivity $\epsilon_{2}=2-i 0.0$. Simple cubic lattice. Squares: prediction from McLachlan equation (Ref. 20).

is quite sharp. We have analyzed the real and imaginary parts of the complex permittivity in terms of the scaling variable $f-f_{c}$. According to the ideas underlying the percolation model, one can obtain the critical exponents $s$ and $t$ by fitting the relations $\epsilon^{\prime} \sim\left|f-f_{c}\right|^{-s}$ and $\epsilon^{\prime \prime} \sim\left(f-f_{c}\right)^{t}$ in the immediate neighbor of the percolation threshold $f_{c}$. Figures 5(a)5 (b) show the data on a $\log -\log$ scale for $\epsilon_{1}=80-i 10^{6}$. The linear part of these graphs yields a value of the critical exponents $s=0.61 \pm 0.05$ and $t=1.23 \pm 0.30$ to be compared with the universality ranges of $0.6-0.7$ for $s$ and $1.5-2$ for $t$ predicted by the percolation model for insulator-conductor mixtures. ${ }^{5}$

\section{B. Effect of crystal lattices}

The results reported up to this point have been obtained from simulation on a sc lattice. The purpose of this section is to examine the effect of crystal lattices on the complex permittivity. In Figs. 6(a)-6(b) we display the real $\epsilon^{\prime}$ and imaginary $\epsilon^{\prime \prime}$ parts of the complex effective permittivity plotted versus $f$ for bec and fcc lattices. To illustrate our point the dielectric constant of the sphere is assumed to be equal to $\epsilon_{1}=80-i 10^{6}$ and that of the remaining volume is $\epsilon_{2}=2-i 0$. The results shown in Figs. 6(a)-6(b) lead to two conclusions. First, the percolation threshold concentration $f_{c}$ depend sensitively on the type of cubic lattice. We cannot estimate $f_{c}$ for bcc and fcc lattices: what we can say is that these values
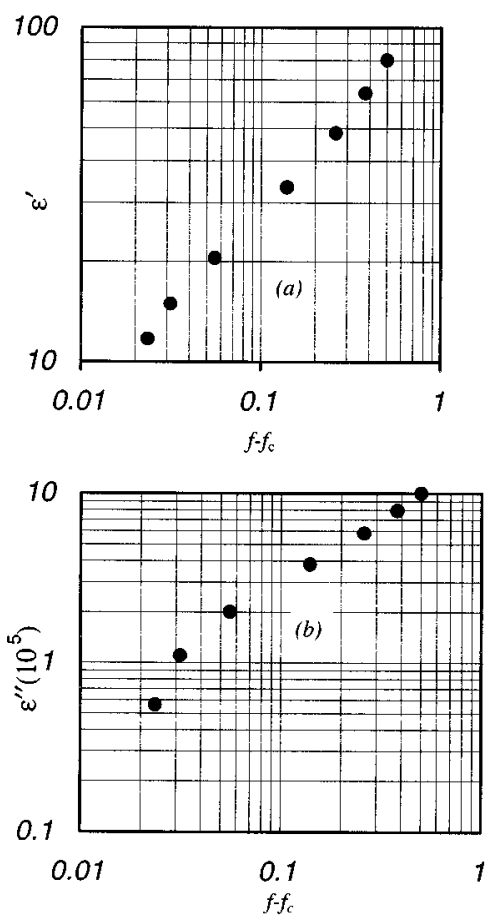

FIG. 5. (a) Real part ( ) of the permittivity $\epsilon^{\prime}$ as a function of the scaling variable $f$ - $f_{c}$; log-log plot above $f_{c}$ with slope $s=0.61 \pm 0.05$. The percolation threshold is $f_{c}=0.50 \pm 0.02$. Inclusions (permittivity $\epsilon_{1}=80-i 10^{6}$ ) are placed in a host matrix material of permittivity $\epsilon_{2}=2-i 0.0$. Simple cubic lattice. (b) Imaginary part $(0)$ of the permittivity $\epsilon^{\prime \prime}$ as a function of the scaling variable $f-f_{c} ; \log \log$ plot above $f_{c}$ with slope $t=1.23 \pm 0.30$. The percolation threshold is $f_{c}=0.50 \pm 0.02$. Inclusions (permittivity $\epsilon_{1}$ $=80-i 10^{6}$ ) are placed in a host matrix material of permittivity $\epsilon_{2}=2-i 0.0$. Simple cubic lattice.

are higher than the corresponding value of the sc lattice, i.e., the higher percolation threshold concentration the higher packing fraction. An explanation for this behavior can probably be ascribed to the fact that the face-centered cubic is the most dense and the simple cubic is the least dense of the three cubic lattices (see Table I). Note that, due to computational limitation, it was not possible to determine the critical exponents $s$ and $t$ from the dependences of $\epsilon^{\prime}$ and $\epsilon^{\prime \prime}$ on the distance from threshold $f-f_{c}$. A second conclusion is that the three different crystal lattices give the same result for $f$ $<0.5$. Thus the effective complex permittivity is insensitive to the geometry of the periodic structure for this range of concentration. However at higher concentrations, we observe some significant differences in both the real and imaginary parts of the permittivity.

\section{Effect of absorption losses}

The purpose of this subsection is to investigate the effect of absorption losses of the dense spheres, i.e., of the ratio $\epsilon_{1}^{\prime \prime} / \epsilon_{1}^{\prime}$. Our results, concerning a sc lattice, are shown in the semilogarithmic plots of Figs. 7(a)-7(b) for $\epsilon_{1}=80-i 10^{n}, n$ $=1,4$ and $\epsilon_{2}=2-i 0$. The data in Fig. 7(a) show that the real parts of the effective permittivity are identical in the range of values of $n$ investigated; whereas the imaginary parts in- 

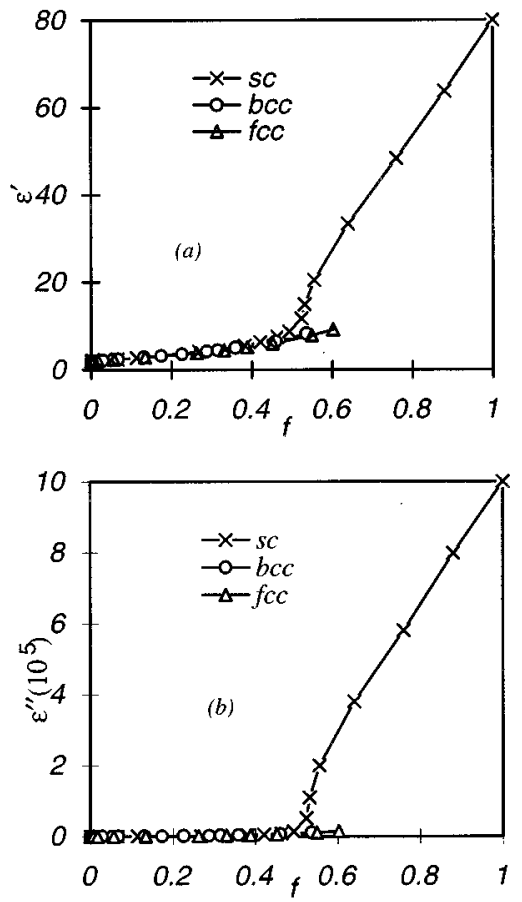

FIG. 6. (a) The real part of the effective permittivity is shown as a function of the volume fraction $f$ of the inclusion phase. Inclusions (permittivity $\epsilon_{1}$ $=80-i 10^{6}$ ) are placed in a host matrix material of permittivity $\epsilon_{2}=2-i 0.0$. Simple cubic lattice $(\times)$, base-centered cubic lattice $(\bigcirc)$, and face-centered lattice $(\triangle)$. (b) The imaginary part of the effective permittivity is shown as a function of the volume fraction $f$ of the inclusion phase. Inclusions (permittivity $\left.\epsilon_{1}=80-i 10^{6}\right)$ are placed in a host matrix material of permittivity $\epsilon_{2}=2-i 0.0$. Simple cubic lattice $(\times)$, base-centered cubic lattice $(\bigcirc)$, and face-centered lattice $(\triangle)$.

crease when the ratio $\epsilon_{1}^{\prime \prime} / \epsilon_{1}^{\prime}$ increases, as displayed in Fig. 7(b). We found a similar behavior for bcc and fcc lattices.

\section{Discussion}

A comparison with computer simulations showed that the McLachlan equation does not accurately reproduce the numerical data over a wide variety of conditions. On the basis of the numerical results described above, an evaluation of the McLachlan's modeling is possible. They may be several reasons for the discrepancy between the predictions of McLachlan equation with our numerical calculations. A useful starting point for discussion of these results comes from the basic assumptions on which McLachlan's modeling rests. Equation (1) rests on a tentative to combine mean field (Bruggeman) theory and percolation theory. The mean field theory of a composite material assumes that the only effect of interactions is to replace the field each dipole feels by an average field. This assumption is not justified for the entire range of volume fractions of the inclusion phase. ${ }^{1,2}$ Further scrutiny of the McLachlan model has led to two additional observations. The first is that the effective medium approach of McLachlan disregards information concerning the detailed structure of the inclusions arrangement in the host medium. The second deals with the fact that the McLachlan equation is written in terms of the conductivities of the two components, a percolation threshold $f_{c}$ and the critical exponent $t$.
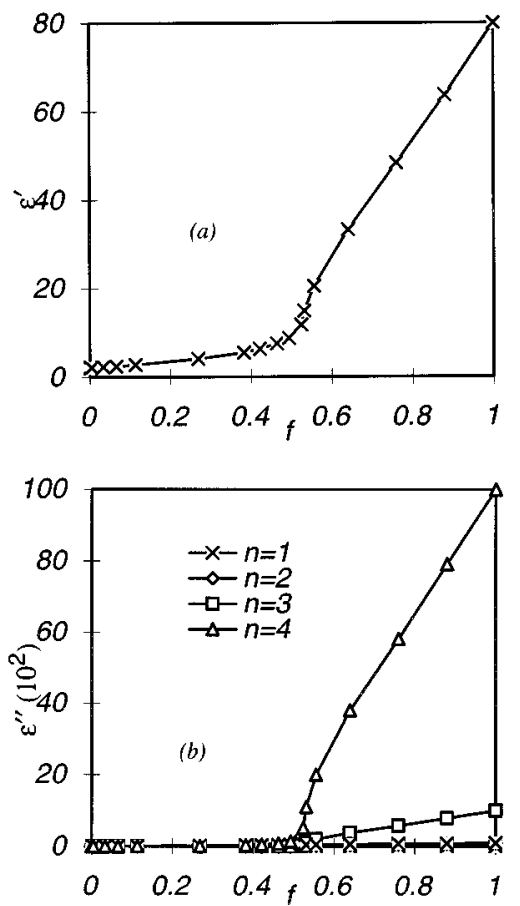

FIG. 7. (a) The real part of the effective permittivity is shown as a function of the volume fraction $f$ of the inclusion phase. Inclusions (permittivity $\epsilon_{1}$ $\left.=80-i 10^{n}, n=1,4\right)$ are placed in a host matrix material of permittivity $\epsilon_{2}$ $=2-i 0.0$. Simple cubic lattice. The values of $\epsilon^{\prime}$ are identical for $n=1,4$. (b) The imaginary part of the effective permittivity is shown as a function of the volume fraction $f$ of the inclusion phase. Inclusions (permittivity $\epsilon_{1}$ $\left.=80-i 10^{n}, n=1,4\right)$ are placed in a host matrix material of permittivity $\epsilon_{2}$ $=2-i 0.0$. Simple cubic lattice.

However we expect that when dealing with permittivity, the mathematical formulation should include the exponent $s$ which determines how $\epsilon^{\prime}$ scales with $f-f_{c}$. It should also be mentioned that the above conclusions are obtained by analysis of materials with finite conductivities.

\section{SUMMARY AND CONCLUDING REMARKS}

We have attempted to show in this article to what extent the McLachlan equation can be used to model the complex effective permittivity of a two-component heterogeneous material, in the quasistatic limit. The technique used has been to compare the results of an ab initio numerical procedure, based on the FE method using the field calculation package FLUX3D which allows the exact values to be computed, to results predicted by this equation. In the first place, a comparison of our numerical data for regular arrays of spherical inclusions, shown in Figs. 3(a)-3(b) and Figs. 4(a)-4(b), with the analytical results obtained with Eq. (1) indicates that McLachlan equation does not provide a good fit for the effective permittivity over the entire range of volume fraction of inclusions. Secondly, the data have been used to examine the relationship between the percolation transition and the permittivity ratio of the two constituents. In this latter context we have illustrated how the percolation threshold changes for cubic lattices. The primary results of this article must be considered the multipole analysis of per- 
mittivity and the numerical evaluation of the percolation threshold that describe critical behavior of the complex effective dielectric constant of a two-component periodic composite material.

This methodology has shown to be robust and accurate for computing the complex effective permittivity of a twocomponent composite material, in a quasistatic approach. It would be very gratifying to see these results tested in real laboratory experiments. Unfortunately, none of the experimental systems are both sufficiently well characterized and studied in enough detail to provide real tests of these numerical data. Nevertheless, if the development of good experimental models is realized, it may be possible in the future to measure some of the effects that were observed here. Research is currently underway to systematically vary parameters like particle shape, spatial arrangement, and size distribution. Such a battery of simulations should make it possible to identify important microstructural information that affect the value of the percolation threshold. These results are relevant for a range of applications such as temperature sensors, corrosion protection devices such as sacrificial anodes and current limiters. ${ }^{30,31}$ Arrays of conducting strips and spheres embedded in dielectric materials are useful microwave devices. ${ }^{32}$ Colloidal crystals which are three-dimensional periodic structures formed from small particles suspended in solution have important technological uses such as optical filters and switches. ${ }^{33}$ The ability to characterize the percolation behavior is important for the design of composite materials having components with finite conductivity because it can be exploited to control complex behavior in these materials. Other properties for which the present results should be directly applicable are the thermal conductivity, the diffusion constant, and the permeability of two-component composites.

${ }^{1}$ R. Landauer, in Electric Transport and Optical Properties of Inhomogeneous Media, edited by J. C. Garland and D. B. Tanner, AIP Conference Proceedings, No. 40 (American Institute of Physics, New York, 1978), p. 2.

${ }^{2}$ Progress in Electromagnetics Research: Dielectric Properties of Heterogenous Materials, edited by A. Priou (Elsevier, New York, 1992), and references therein. No attempt has been made to provide an exhaustive list of articles written on the subject, rather, our references are somewhat eclectic and are chosen to reinforce the main text. Priou's text contains a large reference list, sufficient to whet the appetite of any reader.

${ }^{3}$ E. Yablonovitch, T. J. Gmitter, R. D. Meade, A. M. Rappe, K. D. Brommer, and J. D. Joannopoulos, Phys. Rev. Lett. 67, 3380 (1991); S. L. McCall, P. M. Platzman, R. Dalichaouch, D. Smith, and S. Schultz, ibid. 67, 2017 (1991); W. Robertson, G. Arjavalingan, R. D. Meade, K. D. Brommer, and J. D. Joannopoulos, ibid. 68, 2023 (1992). See also J. D. Joannopoulos, R. Meade, and J. Winn, Photonic Crystals (Princeton University Press, Princeton, 1995).

${ }^{4}$ G. Deutscher, O. Entin-Wohlman, O. Fishman, and Y. Shapira, Phys. Rev. B 21, 5041 (1980).

${ }^{5}$ D. Stauffer and A. Aharony, Introduction to Percolation Theory, 2nd ed.
(Taylor \& Francis, London, 1992). See also S. Kirpatrick, Rev. Mod. Phys. 45, 574 (1973).

${ }^{6}$ For recent reviews on electrical and optical properties, we refer to the Proceedings of the Second International Conference on Electrical and Optical Properties of Inhomogeneous Media [ Physica A 157, (1989)] and to J. P. Clerc, G. Giraud, J. M. Laugier, and J. M. Luck, Adv. Phys. 39, 191 (1990).

${ }^{7}$ A. B. Harris, Phys. Rev. B 28, 2614 (1983).

${ }^{8}$ D. J. Bergman and Y. Imry, Phys. Rev. Lett. 39, 1222 (1977).

${ }^{9}$ D. J. Bergman, Phys. Rev. 43, 377 (1978). See also D. J. Bergman, Ann. Phys. 138, 78 (1982); J. Phys. C 12, 4947 (1979).

${ }^{10}$ D. M. Grannan, J. C. Garland, and D. B. Tanner, Phys. Rev. Lett. 46, 375 (1981).

${ }^{11}$ C. C. Chen and Y. C. Chou, Phys. Rev. Lett. 54, 2529 (1985).

${ }^{12}$ I. Balberg and S. Bozowski, Solid State Commun. 44, 551 (1982).

${ }^{13}$ H. Ottavi, J. Clerc, G. Giraud, J. Roussenq, E. Guyon, and C. D. Mitescu, J. Phys. C 11, 1311 (1978).

${ }^{14}$ Y. Song, T. W. Noh, S. Lee, and J. R. Gaines, Phys. Rev. B 33, 904 (1985).

${ }^{15}$ W. R. Tinga, W. A. G. Voss, and D. F. Blossey, J. Appl. Phys. 44, 3897 (1973).

${ }^{16}$ S. S. Dukhin, Dielectric Properties of Disperse Systems, in Surface and Colloid Science, edited by E. Matievic (Wiley, New York, 1971), Vol. 3, pp. 83-166.

${ }^{17}$ L. K. H. Van Beek, Prog. Dielectr. 7, 69 (1967); R. E. Meredith and C. W. Tobias, Advances in Electrochemistry and Electrochemical Engineering, Vol. 2, edited by C. W. Tobias (Interscience, New York, 1962).

${ }^{18}$ D. J. Bergman and D. Stroud, in Solid State Physics, edited by H. Ehrenreich and D. Turnbull (Academic, New York, 1992), Vol. 46, pp. 178320.

${ }^{19}$ J. V. Mantese, A. L. Micheli, D. F. Dungan, R. G. Geyer, J. Baker-Jarvis, and J. Grosvenor, J. Appl. Phys. 79, 1655 (1996).

${ }^{20}$ D. S. McLachlan, J. Phys. C 20, 865 (1987); see also D. S. McLachlan, Solid State Commun. 72, 831 (1989).

${ }^{21}$ M. A. van Dijk, Phys. Rev. Lett. 55, 1003 (1985).

${ }^{22}$ B. Sareni, L. Krähenbühl, A. Beroual, and C. Brosseau, J. Appl. Phys. 80, 4560 (1996).

${ }^{23}$ G. W. Milton, J. Appl. Phys. 52, 5286 (1981).

${ }^{24}$ B. Sareni, L. Krähenbühl, A. Beroual, and C. Brosseau, J. Appl. Phys. 80, 1688 (1996).

${ }^{25}$ B. Sareni, L. Krähenbühl, A. Beroual, and C. Brosseau, J. Appl. Phys. 81, 2375 (1997).

${ }^{26}$ B. A. Finlaysson, The Method of Weighted Residuals and Variational Principles (Academic, New York, 1972); see also G. Meunier, D. Shen, and J. L. Coulomb, IEEE Trans. Magn. 24, 12 (1988).

${ }^{27}$ W. H. Press, B. P. Flannery, S. A. Teukolsky, and W. T. Vetterling, Numerical Recipes (Cambridge University Press, New York, 1986).

${ }^{28}$ J. P. Calame, A. Birman, Y. Carmel, D. Gershon, B. Levush, A. A. Sorokin, V. E. Semenov, D. Dadon, L. P. Martin, and M. Rosen, J. Appl. Phys. 80, 3992 (1996).

${ }^{29}$ Note that the value $f_{c}=0.16$ used by McLachlan should not be confused with the theoretical prediction of the critical percolation probability for a three-dimensional random closed packing of hard spheres $p_{c}=0.154$ found by Scher and Zallen, J. Chem. Phys. 53, 3759 (1970).

${ }^{30}$ R. Strümpler, J. Appl. Phys. 80, 601 (1996); A discussion of this problem with references is given by J. P. Fitzpatrick, R. B. Malt, and F. Spaepen, Phys. Lett. A 47, 207 (1974).

${ }^{31}$ C. Brosseau, F. Boulic, C. Bourbigot, P. Queffelec, Y. Le Mest, J. Loaec, and A. Beroual, J. Appl. Phys. 81, 882 (1997).

${ }^{32}$ R. E. Collins, Field Theory of Guided Waves (McGraw-Hill, New York, 1960).

${ }^{33}$ A. van Blaaderen, R. Ruel, and P. Wiltzius, Nature (London) 385, 321 (1997). 